\title{
Compositional analysis of polycrystalline hafnium oxide thin films by heavy-ion elastic recoil detection analysis
}

\author{
F.L. Martínez ${ }^{\mathrm{a}, *}$, M. Toledano $^{\mathrm{b}}$, E. San Andrés ${ }^{\mathrm{b}}$, I. Mártil ${ }^{\mathrm{b}}$, G. González-Díaz ${ }^{\mathrm{b}}$, \\ W. Bohne ${ }^{\mathrm{c}}$, J. Röhrich ${ }^{\mathrm{c}}$, E. Strub $^{\mathrm{c}}$ \\ a Departamento de Electrónica y Tecnología de Computadoras, Universidad Politécnica de Cartagena, Campus Universitario Muralla del Mar, \\ E-30202 Cartagena, Spain \\ ${ }^{\mathrm{b}}$ Departamento de Física Aplicada III, Universidad Complutense de Madrid, E-28025 Madrid, Spain \\ ${ }^{c}$ Hahn-Meitner-Institut Berlin, Abteilung SF-4, D-14109 Berlin, Germany
}

Available online 19 January 2006

\begin{abstract}
The composition of polycrystalline hafnium oxide thin films has been measured by heavy-ion elastic recoil detection analysis (HI-ERDA). The films were deposited by high-pressure reactive sputtering (HPRS) on silicon wafers using an oxygen plasma at pressures between 0.8 and 1.6 mbar and during deposition times between 0.5 and $3.0 \mathrm{~h}$. Hydrogen was found to be the main impurity and its concentration increased with deposition pressure. The composition was always slightly oxygen-rich, which is attributed to the oxygen plasma. Additionally, an interfacial silicon oxide thin layer was detected and taken into account. The thickness of the hafnium oxide film was found to increase linearly with deposition time and to decrease exponentially with deposition pressure, whereas the thickness of the silicon oxide interfacial layer has a minimum as a function of pressure at around 1.2 mbar and increases slightly as a function of time. The measurements confirmed that this interfacial layer is formed mainly during the early stages of the deposition process.
\end{abstract}

(C) 2005 Elsevier B.V. All rights reserved.

PACS: 68.37.Lp; 77.84.Bw; 81.15.Cd; 82.80.Yc

Keywords: Hafnium oxide; Elastic recoil detection analysis; Oxygen; Interfacial silicon oxide

\section{Introduction}

There has been much interest in recent times for the application of new dielectrics of high permittivity to the gate structure of CMOS (complementary metal-oxide-semiconductor) transistors $[1,2]$. This is motivated by the reduction of the channel length of these transistors with every new generation, which demands a proportional reduction of the thickness of the gate oxide. However, a limit has been reached (below $65 \mathrm{~nm}$ of channel length) in which no further reduction of the oxide thickness (less than $1.5 \mathrm{~nm}$ for that channel length) seems to be possible, due to excessive tunneling currents that impose intolerable severe power dissipation and heat problems. Therefore, the only alternative is to increase the dielectric constant of the gate dielectric. Nitridation of the silicon oxide

\footnotetext{
* Corresponding author. Tel.: +34 968326 465; fax: +34 968326400 .

E-mail address: Felix.Martinez@upct.es (F.L. Martínez).
}

has been used for this purpose with some success, as well as gate stacks of silicon nitride and silicon oxide [3], but the increase of the dielectric constant achieved in this way is limited by the dielectric constant of silicon nitride $(k=7)$.

Therefore, and in spite of the progress achieved with silicon nitride [4-6] and silicon oxynitride [7-9], it is still necessary to investigate dielectrics with even higher dielectric constant. The problem is that high- $k$ materials such as hafnium oxide $\left(\mathrm{HfO}_{2}\right)$ and zirconium oxide $\left(\mathrm{ZrO}_{2}\right)$ exhibit a tendency to trap electrons. This trapping of charge, especially at the interface with the silicon, generates a shift in threshold voltage during device operation. Mobility degradation of charge carriers is also a problem inherent to the greater polarization characteristic of high- $k$ materials. Much work is being done to overcome these difficulties, given the urgency of the industry to find a solution for the gate dielectric problem. At the moment, the most promising material from a process stability point of view is $\mathrm{HfO}_{2}[10]$. 


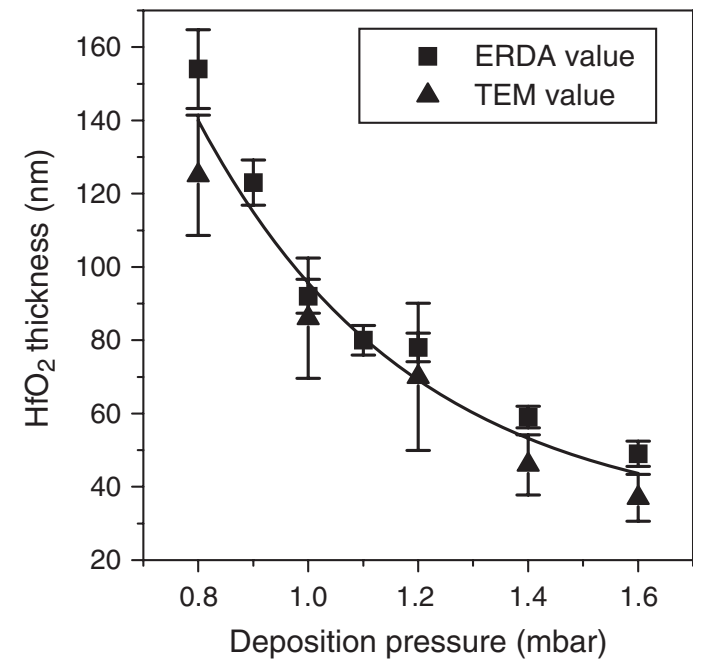

Fig. 1. Thickness of the $\mathrm{HfO}_{2}$ films calculated from the areal density detected by ERDA and from TEM photographs of samples deposited under the same conditions. The deposition pressure is the variable for this series. The line is a fit to the experimental data by an exponential decay curve.

In this article we study the composition of $\mathrm{HfO}_{2}$ films, formed at different deposition parameters by heavy-ion elastic recoil detection analysis (HI-ERDA) [11]. As deposition method we have chosen high pressure reactive sputtering (HPRS), because in this technique the thermalization of plasma molecules reduces the damage of the interface during the growth of the film and we expect that this will result in a lower density of interface states.

\section{Experimental details}

We have deposited $\mathrm{HfO}_{2}$ thin films by HPRS at different deposition pressures and deposition times. The radio frequency power used for the sputtering was $60 \mathrm{~W}$ in all cases, the deposition temperature was $200{ }^{\circ} \mathrm{C}$, and the distance between substrate holder and sputtering target was $2.5 \mathrm{~cm}$. We used targets of $\mathrm{HfO}_{2}$ with a purity of $99.95 \%$. The substrates were ntype silicon wafers $<100>$ oriented with a resistivity of $4.6-$ $5.0 \Omega \mathrm{cm}$. Prior to the deposition procedure the wafers were cut into pieces of $1 \times 1 \mathrm{~cm}^{2}$ and subjected to a standard RCA cleaning procedure [12]. The vacuum in the chamber was $1 \times 10^{-3}$ mbar before the sputtering process was started and the gas used for the plasma was oxygen.

Two sample series were deposited. In the first one we varied the deposition pressure from 0.8 to $1.6 \mathrm{mbar}$, with a fixed deposition time of $3 \mathrm{~h}$. In the second series we kept constant the deposition pressure at $1.2 \mathrm{mbar}$ and we reduced the deposition time from $3 \mathrm{~h}$ to half an hour. In this study, we will concentrate on the measurements of composition and thickness by HI-ERDA, and we will compare the thickness values with those obtained from cross-sectional photographs by transmission electron microscopy (TEM). One additional sample with a deposition time of 15 min was investigated by TEM only.

The HI-ERDA measurements were performed at the IonenStrahl-Laboratorium (ISL) of the Hahn-Meitner-Institut in Berlin. A beam of $350 \mathrm{MeV}$ Au ions was used. The recoiled sample atoms were detected by a mass and energy dispersive spectrometer, for details see [13]. The total ion dose was in the order of $10^{11}$ atoms, as measured with good accuracy $(\sim 2 \%)$ by a transmission Faraday cup [14], while the ion fluence in atoms per square centimeter suffered from the uncertainty in the determination of the irradiated spot area $\left(\sim 2 \times 5 \mathrm{~mm}^{2}\right)$. This results in an ion fluence in the order of $10^{12}$ atoms $/ \mathrm{cm}^{2}$, which is low enough to minimize preferential effusion effects of light atoms, taking place at higher ion fluence [15].

\section{Results and discussion}

\subsection{Influence of deposition pressure}

We have analyzed seven samples deposited at deposition pressures between 0.8 and 1.6 mbar. The deposition time was $3 \mathrm{~h}$ for all of them. Figs. 1 and 2 show the thickness of the $\mathrm{HfO}_{2}$ film and the interfacial layer, which has been identified as $\mathrm{SiO}_{2}$ by infrared spectroscopy. These values are obtained from the TEM photographs and, in the case of the $\mathrm{HfO}_{2}$ films, also from the ERDA areal density (number of atoms per unit area) using the bulk density for pure $\mathrm{HfO}_{2}$ [16]. A remarkable agreement is observed.

In addition to the areal density, the ERDA measurements provide the atomic concentration of each constituent of the films. In Table 1 we include some of these results. The ion fluences for all ERDA measurements were low (in the order of $10^{12} \mathrm{at} / \mathrm{cm}^{2}$ ), so that effusion effects, typical for high doses, were only observed for $\mathrm{H}$ and in some cases for $\mathrm{C}$ [15]. An extrapolation procedure to zero dose was applied in the evaluation of the $\mathrm{H}$ and $\mathrm{C}$ concentrations. The summed content of other impurities detected in very small concentrations are also included in the table, as well as the ratio of the oxygen and hafnium concentrations (stoichiometry). The uncertainty of this ratio is about $5 \%$, due to the counting statistics and, especially in the case of the thinnest films, due to the problem of subtracting the $\mathrm{O}$ fraction coming from the $\mathrm{SiO}_{2}$ interfacial

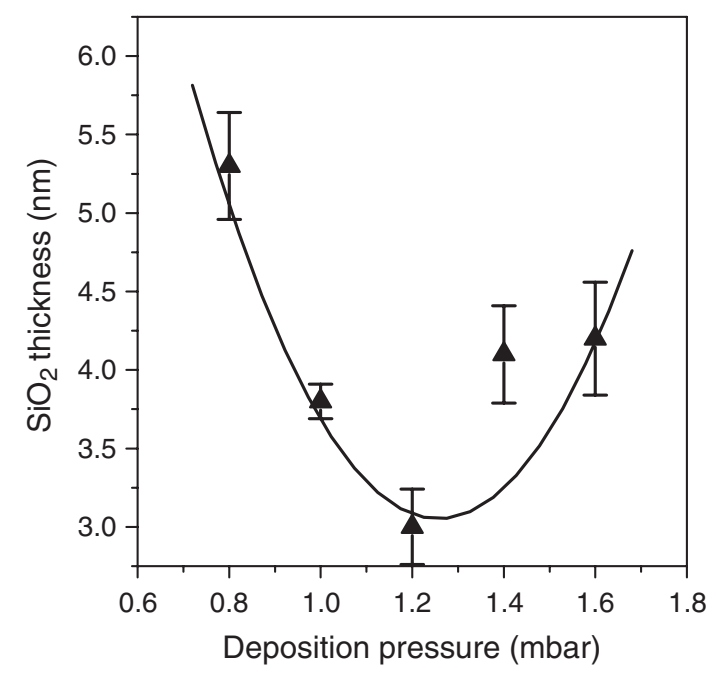

Fig. 2. Thickness of the $\mathrm{SiO}_{2}$ interfacial layer as a function of deposition pressure. Values obtained from TEM photographs are shown. The continuous curve is a parabolic fit to the experimental data points. 
Table 1

Summary of composition results by ERDA for the sample series deposited as a function of pressure

\begin{tabular}{|c|c|c|c|c|c|c|c|}
\hline Deposition pressure (mbar) & 0.8 & 0.9 & 1.0 & 1.1 & 1.2 & 1.4 & 1.6 \\
\hline Ion fluence $\left(\times 10^{12} \mathrm{at} / \mathrm{cm}^{2}\right)$ & 8.51 & 6.09 & 6.41 & 5.65 & 6.68 & 6.77 & 6.78 \\
\hline $\mathrm{Hf}($ at.\%) & 30.73 & 30.10 & 30.36 & 29.40 & 28.50 & 29.40 & 30.59 \\
\hline $\mathrm{O}($ at. $\%)$ & 65.00 & 62.47 & 61.44 & 63.56 & 62.57 & 63.69 & 62.30 \\
\hline Other impurities (at.\%) (B, C, N, F, Zr) & 1.09 & 1.93 & 3.1 & 3.04 & 3.63 & 0.91 & 1.11 \\
\hline Stoichiometry: $[\mathrm{O}] /[\mathrm{Hf}]$ ratio & 2.12 & 2.07 & 2.02 & 2.16 & 2.19 & 2.17 & 2.04 \\
\hline
\end{tabular}

The uncertainty of the $[\mathrm{O}] /[\mathrm{Hf}]$ ratio is about $5 \%$.

layer. In all simulation calculations we used the thickness for this layer according to the TEM results and assuming pure $\mathrm{SiO}_{2}$.

All measured values are slightly oxygen-rich. We observe that the hafnium concentration has a weak minimum at a deposition pressure of about 1.2 mbar resulting in a composition ratio of almost 2.2. This excess of oxygen is attributed to the incorporation of oxygen from the oxygen plasma during the growth process. It must be noted that a hafnium atom is relatively big compared with silicon, and the $\mathrm{Hf}-\mathrm{O}$ bonds are longer than the $\mathrm{Si}-\mathrm{O}$ bonds of silicon dioxide [17], so the hafnium oxide layers inherently have much more strain than silicon dioxide. Therefore, the incorporation of oxygen atoms from the plasma tends to relieve this stress. Hf atoms can have higher oxygen coordination than silicon, so an excess of oxygen can be easily accommodated in the lattice.

The main impurity in the samples is hydrogen, which is not pumped completely by the vacuum system of the sputtering machine. Other impurities of light atoms such as $\mathrm{B}, \mathrm{C}, \mathrm{N}$ and $\mathrm{F}$ are also detected. $\mathrm{Zr}$ is a common impurity in hafnium oxide, and it originates from the cathode. The samples deposited between 1.0 and 1.2 mbar are the ones with the higher content of impurities, mainly due to a particularly high content of $\mathrm{C}$.

As shown in Fig. 1, the variation of the thickness of the $\mathrm{HfO}_{2}$ films follows a very clear trend with the deposition pressure. This trend can be fitted by an exponential decay. Additionally, the very good agreement between the values of ERDA and TEM confirms the validity of the data. The kinetics of the sputtering process clearly become more inefficient as the pressure is increased. This is due to the reduction of the mean free path of the plasma molecules at higher pressure. As the collisions between gas molecules become more frequent, the energy with which they bombard the sputtering target is reduced and the growth velocity is reduced.

The interfacial $\mathrm{SiO}_{2}$ layers, whose thickness values are shown in Fig. 2, are formed due to the oxidation of the silicon surface by the oxygen plasma during the first stages of the sputtering process, as we will see in the next section. The thickness is very small, but nevertheless it has been taken into account in the evaluation procedure of the ERDA spectra. The decreasing trend for deposition pressures up to $1.2 \mathrm{mbar}$ is reversed at higher values of deposition pressure, resulting in a minimum thickness for a value close to 1.2 mbar. This might be explained by two competing forces. On the one hand, the already mentioned decrease in the mean free path of the oxygen molecules of the plasma at higher gas pressure results in a decrease of the energy with which these molecules bombard not only the cathode, but also the substrate. Hence, both the sputtering process and the oxidation of the substrate become more inefficient as the gas pressure is increased. On the other hand, an increase of the gas pressure can also favor the diffusion rate of the gas molecules through the growing $\mathrm{HfO}_{2}$ film. The oxygen molecules arriving at the interface can continue to promote the growth of the $\mathrm{SiO}_{2}$ interfacial layer at the same time that the $\mathrm{HfO}_{2}$ film is growing. As the diffusion rate is proportional to the oxygen pressure, the growth velocity of the $\mathrm{SiO}_{2}$ layer is expected to increase at high deposition pressures.

\subsection{Influence of deposition time}

The main goal of our study is to determine the optimum deposition conditions for $\mathrm{HfO}_{2}$ films with our HPRS plasma system. Specifically, we are interested in the deposition conditions which result in the optimum properties for the application of the dielectric to the gate structure of CMOS transistors. For this purpose, it is necessary to minimize the equivalent oxide thickness (EOT) of the gate dielectrics. Clearly, the presence of a silicon oxide layer in this structure limits the minimum achievable EOT, because in no case it can be smaller than this interfacial $\mathrm{SiO}_{2}$ layer. For this reason we have chosen the pressure of $1.2 \mathrm{mbar}$ for the next part of our study, based on the results of the previous section, which gave a minimum thickness of $\mathrm{SiO}_{2}$ for this pressure.

A summary of composition results obtained by ERDA is shown in Table 2 for samples deposited between 0.5 and $3 \mathrm{~h}$ in increments of $0.5 \mathrm{~h}$. Again, all samples analyzed are slightly oxygen-rich, with oxygen to hafnium ratios between 2.03 and 2.25. The same impurities as for the previous series were detected with similar concentrations. The most interesting result is the areal density of each layer, which converted into

Table 2

Composition results obtained by ERDA for the sample series deposited as a function of time

\begin{tabular}{lrrrrrr}
\hline Deposition time (h) & 0.5 & 1.0 & 1.5 & 2.0 & 2.5 & 3.0 \\
\hline Ion fluence $\left(\times 10^{12} \mathrm{at} / \mathrm{cm}^{2}\right)$ & 5.76 & 5.10 & 4.01 & 4.02 & 3.31 & 6.68 \\
$\mathrm{Hf}($ at.\%) & 29.32 & 30.6 & 29.70 & 30.77 & 31.57 & 28.50 \\
$\mathrm{O}($ at.\%) & 66.00 & 63.91 & 60.88 & 64.00 & 64.00 & 62.57 \\
$\mathrm{H}($ at.\%) & 4.00 & 4.70 & 6.60 & 4.22 & 3.30 & 5.30 \\
Other impurities (at.\%) (B, C, N, F, Zr) & 0.68 & 0.79 & 2.82 & 1.01 & 1.13 & 3.63 \\
Stoichiometry: $[\mathrm{O}] /[\mathrm{H}]$ ratio & 2.25 & 2.09 & 2.05 & 2.08 & 2.03 & 2.19
\end{tabular}

Uncertainties are in the order of $\sim 7 \%$. 


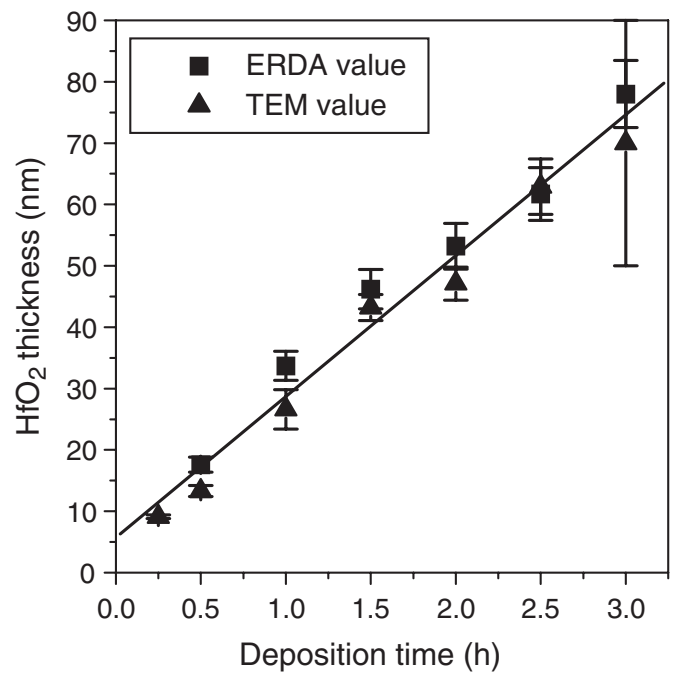

Fig. 3. Thickness of the $\mathrm{HfO}_{2}$ films as a function of the deposition time. Values obtained from the ERDA areal density and from TEM cross-sections are shown. The solid line is a linear fit.

thickness by using the bulk value for the density of $\mathrm{HfO}_{2}$ are plotted in Fig. 3.

The thickness of the $\mathrm{HfO}_{2}$ film displayed in Fig. 3 shows a linear dependence with deposition time. As in the previous section, the values of thickness obtained by ERDA and those obtained from the cross-section TEM photographs are in very good agreement. The electrical characteristics of these films are now in the process of being evaluated, but if we assume a dielectric constant of 23 [10] we can calculate the equivalent oxide thickness (the thickness of a silicon dioxide film that would have the same capacitance). As a result we obtain that the thinnest of our films (10 nm, see Fig. 3) would have an equivalent oxide thickness of $1.7 \mathrm{~nm}$, which is close to the value required for present-day $65 \mathrm{~nm}$ channel devices.

However, the problem is that below this $\mathrm{HfO}_{2}$ there is an interfacial layer of $\mathrm{SiO}_{2}$. Fig. 4 shows its thickness as a function of deposition time, obtained from the TEM photographs. The thickness of this layer increases as a function of deposition time, abruptly in the first half hour and moderately for longer deposition times. This indicates that this layer is formed mainly during the first instants of the deposition process by the oxidizing action of the plasma gas, but its growth continues during the remaining deposition process at a lower rate due to the diffusion of oxygen through the growing $\mathrm{HfO}_{2}$ film. The thickness of this layer limits the minimum achievable EOT, because its value must be added to the EOT of the $\mathrm{HfO}_{2}$ film. For example, for the thinnest of the films deposited in this study the total EOT would be $4.8 \mathrm{~nm}(1.7 \mathrm{~nm}$ for the EOT of the $\mathrm{HfO}_{2}$ layer plus $3.1 \mathrm{~nm}$ for the $\mathrm{SiO}_{2}$ layer), which is far from the values demanded by the industry for the next generations of CMOS (EOT $<1.5 \mathrm{~nm}$ for gate lengths below $65 \mathrm{~nm}$ ).

It is therefore necessary to reduce the thickness of this interfacial $\mathrm{SiO}_{2}$ layer. For this purpose we have started to work on a modification of our HPRS system in order to perform the initial stages of the growth process with an argon plasma instead of an oxygen plasma. In this way we hope to avoid the rapid initial oxidation of the surface. After the first few minutes of sputtering, we will continue the process with oxygen because we believe that a small incorporation of oxygen atoms from the plasma gas tends to reduce the inherent strain of the $\mathrm{HfO}_{2}$ film, as we pointed out in the previous section.

\section{Conclusions}

We have studied the composition of $\mathrm{HfO}_{2}$ thin films deposited by HPRS under different conditions of gas pressure and deposition time. The atomic concentration of each constituent element and the areal density of each layer were obtained from HI-ERDA measurements. Hydrogen was found to be the main impurity, although traces of other light atoms such as B, C, N and F were also detected. The stoichiometry was slightly oxygen-rich in all cases, which is attributed to incorporation of oxygen atoms from the plasma gas.

From the HI-ERDA areal density we calculated the film thickness and found a good agreement with the values obtained from cross-section TEM photographs. The presence of a thin $\mathrm{SiO}_{2}$ interfacial layer was taken into account. As a function of pressure, the thickness of the $\mathrm{HfO}_{2}$ film shows an exponential decay, which is attributed to the less efficient process of sputtering of the cathode at high gas pressures, whereas the $\mathrm{SiO}_{2}$ thickness shows an initial decrease as a function of gas pressure, but an increase at higher gas pressures, probably due to the higher diffusion rate of oxygen molecules through the growing $\mathrm{HfO}_{2}$ film. As a function of time, the thickness of the $\mathrm{HfO}_{2}$ film increases linearly, while the $\mathrm{SiO}_{2}$ interfacial layer is mostly formed during the early stages of the deposition process.

Based on the results of this study, we have chosen 1.2 mbar as the optimum deposition pressure, because it results in the minimum thickness of the interfacial $\mathrm{SiO}_{2}$ film, and for future experiments we have decided to avoid the use of oxygen during

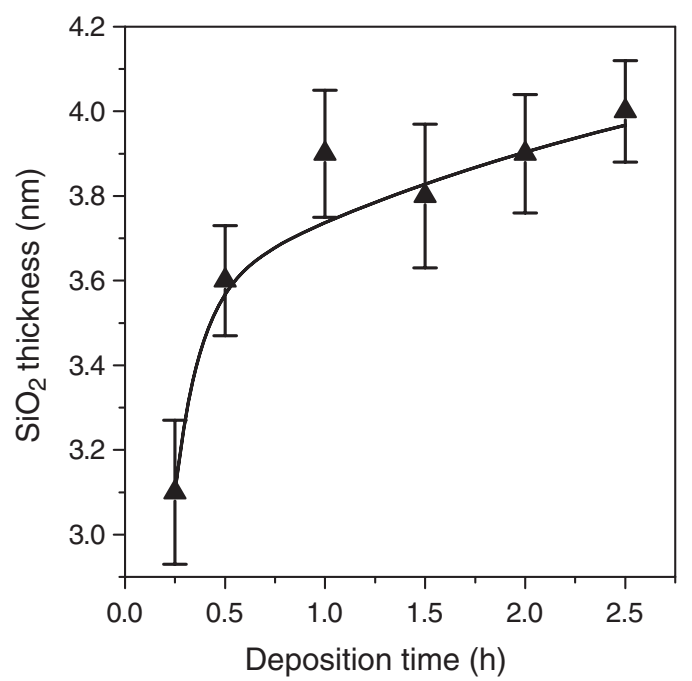

Fig. 4. The thickness of the $\mathrm{SiO}_{2}$ interfacial layer as a function of the deposition time. Thickness values are obtained from TEM photographs. The solid line is an exponentially asymptotic fit. 
the early stages of film growth in order to minimize the thickness of this interfacial layer.

\section{Acknowledgements}

This work was made possible thanks to a mobility grant of the Secretaría de Estado de Educación y Universidades of the Spanish Ministry of Education and Science. It was also supported by the research project TEC2004/1237 of the same Ministry. Special thanks are given to the ISL for hosting this work.

\section{References}

[1] G.D. Wilk, R.M. Wallace, J.M. Anthony, J. Appl. Phys. 89 (2001) 5243.

[2] A.I. Kingon, J.P. Maria, S.K. Streiffer, Nature 406 (2000) 1032.

[3] D.A. Buchanan, IBM J. Res. Develop. 43 (1999) 245.

[4] F.L. Martínez, R. Ruiz-Merino, A. del Prado, E. San Andrés, I. Mártil, G. González-Díaz, C. Jeynes, N.P. Barradas, L. Wang, H.S. Reehal, Thin Solid Films 459 (2004) 203.

[5] I. Mártil, A. del Prado, E. San Andrés, G. González-Díaz, F.L. Martínez, J. Appl. Phys. 94 (2003) 2642.
[6] E. San Andrés, A. del Prado, I. Mártil, G. González-Díaz, F.L. Martínez, J. Vac. Sci. Technol. 21 (2003) 1306.

[7] A. del Prado, E. San Andrés, I. Mártil, G. González-Díaz, D. Bravo, F.J. López, M. Fernández, F.L. Martínez, J. Appl. Phys. 94 (2003) 1019.

[8] A. del Prado, E. San Andrés, I. Mártil, G. González-Díaz, D. Bravo, F.J. López, W. Bohne, J. Röhrich, B. Selle, F.L. Martínez, J. Appl. Phys. 93 (2003) 8930.

[9] A. del Prado, E. San Andrés, F.L. Martínez, I. Mártil, G. González-Díaz, W. Bohne, J. Röhrich, B. Selle, M. Fernández, Vacuum 67 (2002) 507.

[10] Y.S. Lin, R. Puthenkovilakam, J.P. Chang, Appl. Phys. Lett. 81 (2002) 2041.

[11] J.C. Barbour, B.L. Doyle, in: J.R. Tesmer, M. Nastasi (Eds.), Handbook of Modern Ion Beam Materials Analysis, Materials Research Society, Pittsburgh, 1995, p. 83.

[12] W. Kern, D. Puotinen, RCA Rev. 31 (1970) 187.

[13] W. Bohne, J. Röhrich, G. Röschert, Nucl. Instrum. Methods Phys. Res., B Beam Interact. Mater. Atoms 136-138 (1998) 633.

[14] W. Bohne, S. Hessler, G. Röschert, Nucl. Instrum. Methods Phys. Res., B Beam Interact. Mater. Atoms 113 (1996) 78.

[15] W. Bohne, W. Fuhs, J. Röhrich, B. Selle, G. González-Díaz, I. Mártil, F.L. Martínez, A. del Prado, Surf. Interface Anal. 30 (2000) 534.

[16] R.C. Weast, M.J. Astle, W.H. Beyer (Eds.), CRC Handbook of Chemistry and Physics, CRC Press, Boca Ratón, 1987.

[17] A. Kawamoto, J. Jameson, P. Griffin, K. Cho, R. Dutton, IEEE Electron Device Lett. 22 (2001) 14. 